\title{
Funções de Estímulos Pré-experimentais na Formação de Classes de Equivalência
}

\author{
Verônica Bender Haydu, * (D), Carolyne Tomaz de Aquino", \\ Larissa Botelho Gaça ${ }^{2}$ (D), \& Gerson Yukio Tomanari ${ }^{3}$ (D) \\ ${ }^{1}$ Universidade Estadual de Londrina, Londrina, PR, Brasil \\ ${ }^{2}$ Universidade Federal de São Paulo, São Paulo, SP, Brasil \\ ${ }^{3}$ Universidade de São Paulo, São Paulo, SP, Brasil
}

\begin{abstract}
RESUMO - Relações condicionais pré-experimentais que eliciam respostas emocionais, como ciúme, podem afetar a formação de classes de equivalência? Haveria diferenças entre homens e mulheres? Para abordar essas questões, 19 participantes, de ambos os sexos, foram expostos a treinos e testes para a formação de quatro classes de equivalência, uma delas constituída pelo nome do(a) namorado(a), o nome de mulher/homem desconhecida(o) e duas figuras não-familiares. Após os testes, aplicou-se a Escala de Ciúme Romântico. Os participantes classificados com maior grau de ciúme não formaram as classes de equivalência, independentemente de serem homens ou mulheres. Sugere-se que relações préexperimentais com funções afetivas interferiram na formação das classes de equivalência em que o nome do namorado(a) deveria ser relacionado ao nome de desconhecido(a).
\end{abstract}

PALAVRAS-CHAVE: classes de equivalência, ciúme romântico, relações condicionais, escolha de acordo com modelo

\section{Pre-experimental Stimuli Functions in Equivalence Class Formation}

\begin{abstract}
Can pre-experimental conditional relations that elicit emotional responses, such as jealousy, affect the formation of equivalence classes? Would there be differences between men and women? To address these questions, 19 participants of both sexes were exposed to training and testing of the formation of four equivalence classes, one consisting of the boyfriend's/girlfriend's name, an unknown woman's/man's name, and two unfamiliar figures. After the tests, the Romantic Jealousy Scale was applied. Participants classified as having a higher degree of jealousy did not form equivalence classes regardless of whether they were men or women. It is suggested that pre-experimental relations with affective functions interfere in the formation of equivalence classes in which the boyfriend's/girlfriend's name should be related to the unknown woman's/man's name.
\end{abstract}

KEYWORDS: equivalence classes, romantic jealousy, conditional relations, matching-to-sample

O conceito de classes de estímulos equivalentes proposto por Sidman e Tailby (1982) tem fundamentado as pesquisas sobre fenômenos comportamentais complexos e o desenvolvimento de tecnologias comportamentais (para revisão ver Rehfeldt, 2011; Sidman, 1994), bem como as investigações sobre o efeito de variáveis envolvidas em aspectos que causam problemas psicológicos (e. g., Adcock et al., 2010; Munnelly, Martin, Dack, Zedginidze, $\&$ McHugh, 2014). Não há dúvida de que estudar como as classes de equivalência são formadas, mantidas e modificadas tem relevância científica e social, em particular no contexto clínico e educacional.

A formação de classes de estímulos equivalentes pode ser demonstrada a partir do ensino de, pelo menos, duas relações condicionais entre estímulos com um elemento em comum, seguido por testes de relações emergentes (Sidman, 2000). O ensino das relações condicionais, geralmente, é feito por meio do procedimento de escolha de acordo com o modelo (do inglês matching to sample [MTS]). Esse procedimento consiste na apresentação de um estímulo condicional

*E-mail: veronicahaydu@gmail.com

- Submetido: 10/10/2016; Revisado: 07/06/2017; Aceito: 12/03/2018. 
(estímulo-modelo) e dois ou mais estímulos discriminativos diferentes (estímulos de comparação), e na apresentação de uma consequência reforçadora para a resposta de escolher o estímulo de comparação que corresponde ao estímulomodelo definido como correto. Por exemplo, para ensinar as relações condicionais pré-requisito para a formação de duas classes de equivalência, com três estímulos cada, deve-se: na presença do estímulo-modelo A1, reforçar a escolha de B1, dentre dois estímulos de comparação (B1 e B2); e, diante do estímulo-modelo $\mathrm{B} 1$, reforçar a escolha de $\mathrm{C} 1$, dentre dois estímulos de comparação $(\mathrm{C} 1$ e $\mathrm{C} 2)$. Dessa maneira, são ensinadas as relações A1-B1 e B1-C1, devendo ser treinadas, também, as relações A2-B2 e B2-C2. Após essa sequência de treinos, são testadas as relações emergentes de reflexividade, de simetria e de transitividade. A reflexividade caracteriza-se pela relação de identidade entre os estímulos, como a escolha do estímulo de comparação A1 diante do estímulo-modelo A1. A simetria caracteriza-se pela reversibilidade funcional entre o estímulo-modelo e o de comparação, ou seja, escolher A1 diante do estímulo-modelo $\mathrm{B} 1$ deve emergir. A transitividade e a transitividade simétrica são demonstradas na escolha do estímulo $\mathrm{C} 1$ diante de $\mathrm{A} 1$, e na escolha de A1 diante de $\mathrm{C} 1$, respectivamente. Se as relações com as propriedades de reflexividade, simetria, transitividade e transitividade simétrica emergirem, diz-se que há equivalência entre os estímulos daquela classe (Sidman, 2000).

Uma característica das classes de equivalência é o fato de ocorrer a transferência de funções de estímulo entre seus membros, ou seja, alterações na função de um dos membros da classe afetam todos os demais, sem que tenha havido um emparelhamento direto entre estímulos (condicionamento do tipo S-S). Por exemplo, Dougher, Augustson, Markham, Greenway e Wulfert (1994, Estudo 1) demonstraram esse processo em um estudo com oito estudantes universitárias, no qual foram treinadas relações condicionais entre estímulos que levaram à formação de duas classes de estímulos com quatro membros cada (A1B1C1D1 e A2B2C2D2). Em seguida, um choque elétrico de baixa intensidade foi emparelhado com o estímulo B1 (o estímulo B2 não foi emparelhado com o choque). Medidas da resposta da condutibilidade galvânica da pele foram registradas. Verificou-se que sete das oito participantes apresentaram alteração da resposta galvânica da pele na presença de todos os estímulos da Classe 1, da qual fazia parte o estímulo B1, mas não na presença dos estímulos da Classe 2. Esses resultados foram interpretados como indicativos de transferência da função eliciadora do estímulo diretamente pareado ao choque aos demais estímulos que faziam parte da classe de estímulos equivalentes, mas que não foram emparelhados diretamente ao choque.

A possibilidade de formação de classes de equivalência e de haver transferência de funções entre os estímulos contribuiu para que o paradigma da equivalência de estímulos passasse a ser uma forma de explicar o comportamento simbólico (de Rose \& Bortoloti, 2007; Postalli, Schmidt, Nakachima, \& de Souza, 2013; Sidman, 1990). Por essa razão, esse paradigma vem sendo usado em pesquisas sobre formação de atitudes, como preconceitos raciais e religiosos (e.g., de Carvalho \& de Rose, 2014; Dixon \& Lemke, 2007; Watt, Keenan, Barnes, \& Cairns, 1991), autoavaliação de eficácia e autorreferência (Dack, McHugh, \& Reed, 2012; Merwin \& Wilson, 2005), exclusão social (Munnelly et al., 2014), jogo patológico (Dixon, Bihler, \& Nastally, 2011), revelação de experiências traumáticas (Keenan, McGlinchey, Fairhurst, \& Dillenburger, 2000) e outros comportamentos clinicamente relevantes (Adcock et al., 2010; Haydu, Gaça, Cognetti, Costa, \& Tomanari, 2015; Leslie, Ulster-Jordanstown, Tierney, Robinson, \& Keenan, 1993; Plaud, 1995; Plaud, Gaither, Franklin, Weller, \& Barth, 1998). Em diversos estudos dessa literatura (e.g., Merwin \& Wilson, 2005), eventos da história pré-experimental, relevantes para o fenômeno abordado, são mensurados, o que permite avaliar como estímulos (e.g., nomes ou figuras) com determinadas funções previamente estabelecidas passam a fazer parte ou a interferir na formação de classes de equivalência estabelecidas experimentalmente.

Um estudo que demonstrou que funções de estímulos estabelecidas pré-experimentalmente podem interferir na formação de classes de equivalência foi desenvolvido por Plaud (1995). Participaram desse experimento 51 mulheres que foram expostas a treinos de relações condicionais por meio de MTS e aos subsequentes testes de relações emergentes, envolvendo dois tipos de estímulos: nomes de flores (fear-irrelevant) e nomes de cobras ou serpentes (fear-relevant). No final do procedimento, foram aplicados o Snake Questionnaire e 30 questões sobre medo de cobras. $\mathrm{O}$ número de blocos de treino e de teste necessário para a formação de classes de equivalência foi maior quando os estímulos eram nomes de cobras ou de serpentes do que quando os estímulos eram nomes de flores. Além disso, verificou-se que os dados obtidos pelas medidas de relato verbal do medo de cobras eram preditores do número de blocos requeridos para a formação das classes de equivalência.

No estudo desenvolvido por Haydu et al. (2015) também foram usados nomes com funções pré-experimentais como estímulos a serem relacionados de forma arbitrária. $\mathrm{O}$ uso de nomes do repertório dos participantes em vez de estímulos arbitrários, como é comum em estudos de equivalência de estímulos, permite que relações condicionais extraexperimentais sejam trazidas para as situações experimentais, conforme apontaram Clayton e Hayes (2007), sem ser necessário o desenvolvimento de uma história específica. No estudo de Haydu et al., foram utilizados o nome do(a) namorado(a) e nomes de homens e/ou de mulheres desconhecidos(as) do participante. O objetivo do estudo foi avaliar se relações de equivalência seriam afetadas pela condição de os participantes terem que relacionar o nome do(a) namorado(a) com o nome 
de mulher/homem desconhecido(a), correlacionando o desempenho deles nos testes de equivalência com escores obtidos na Escala de Ciúme Romântico - ECR (Ramos, Yazawa, \& Salaza, 1994). A função pré-experimental do nome do(a) namorado(a) foi inferida a partir da ECR, que foi aplicada na última fase do procedimento, assim, como no estudo de Plaud (1995). Verificou-se que houve correlação entre os escores obtidos pelos participantes na ECR e o desempenho nos testes de relações emergentes (i.e., maior grau de ciúme foi acompanhado por pior desempenho no teste das relações emergentes). Entretanto, nesse estudo, apenas um participante apresentou baixo escore de ciúme; esse participante era do sexo masculino e apresentou $100 \%$ de acertos no teste de equivalência. Os demais participantes (16), todos do sexo feminino, apresentaram escores entre moderado e intenso. Dessa forma, esse estudo não somente envolveu uma faixa pequena de variação dos dados da ECR, como também não permitiu avaliar, inquestionavelmente, se a emergência de classes de equivalência foi afetada pelo grau de ciúme e/ou pelo sexo do participante. Considerando que características evolutivas podem determinar diferenças entre os sexos no tocante ao ciúme (Buss, 1994), o presente estudo recrutou um número maior de participantes do sexo masculino, o que poderia aumentar a variação no grau de ciúme entre participantes e permitir uma avaliação mais adequada do papel de ambas as variáveis (ciúme e sexo) na formação de classes de equivalência.

Assim, o objetivo do presente estudo foi replicar sistematicamente aquele desenvolvido por Haydu et al. (2015) de modo a permitir: (a) analisar o efeito de estímulos com valor afetivo pré-experimentalmente estabelecido, inferido a partir da Escala de Ciúme Romântico, na formação de classes de estímulos equivalentes, (b) analisar a formação de classes de equivalência em função do sexo dos participantes. A escolha do ciúme [comportamento emocional caracterizado como sendo um conjunto complexo de comportamentos respondentes e operantes interligados (Costa, 2009)] como tema da análise foi feita devido às implicações que os estudos sobre equivalência de estímulos e transferência de função têm para a compreensão de fenômenos clinicamente relevantes, com foi apontado por Dougher (1998). Além disso, esse tema, bem como o estímulo "nome do namorado(a)" foi selecionado porque Costa et al. (2014) demonstraram que os participantes relacionaram a palavra "ciúme" à palavra "amor", permitindo sugerir que há relações condicionais entre esses nomes.

Com base nos dados apresentados por Costa et al. (2014) e em observações não sistemáticas, considerou-se que o nome do(a) namorado(a) e o nome da(o) própria(o) participante, provavelmente, são frequentemente emparelhados no cotidiano e que essa relação é fortalecida por reforços positivos. Assim, para ampliar a análise quanto ao tipo de estímulo empregado com função pré-experimental, um dos nomes de pessoa desconhecida (usado no estudo de Haydu et al., 2015) foi substituído pelo nome do(a) próprio(a) participante. Isso foi realizado de forma que, no treino de relações condicionais, o nome do participante nunca era relacionado ao nome do namorado(a), mas sim a um nome desconhecido; o nome do namorado(a) também era relacionado a um nome desconhecido. Esse tipo de arranjo experimental permite avaliar a possível dificuldade no estabelecimento de novas relações condicionais inconsistentes com essa história de reforço (cf., Santos, Mizael, \& de Rose, 2016). Além da modificação feita em relação à inclusão do nome do(a) próprio(a) participante, 0 número de tentativas de teste das relações de transitividade e de transitividade simétrica foi aumentado para que todas as relações condicionais fossem testadas mais de uma vez.

Em suma, a justificativa do presente estudo reside em investigar o ciúme, uma instância comportamental clinicamente relevante, com base em evidências que permitem inferir que relações pré-experimentais inconsistentes com relações estabelecidas experimentalmente podem interferir na formação das classes de equivalência envolvendo essas relações, conforme apontam os resultados de estudos como o de Dixon e Lemke (2007) e Haydu et al. (2015), dentre outros. As relações pré-experimentais em diversos estudos dessa natureza são mensuradas por meio de escalas de autorrelato (e.g., Adcock et al., 2010; Merwin \& Wilson, 2005; Plaud, 1995), uma vez que permitem inferir o tipo de relação pré-experimental entre os estímulos a serem relacionados, a partir de descrições de sentimento e atitudes em relação a esses estímulos ( $c f$., de Wulfert, Greenway, Farkas, Hayes \& Dougher, 1994). A ECR era aplicada após a realização do procedimento de formação e teste das relações emergentes reduzindo a possibilidade de os participantes discriminarem que se tratava de um estudo relacionado ao ciúme.

\section{MÉTODO}

\section{Participantes}

Foram selecionados 20 estudantes universitários, matriculados em diferentes cursos de uma universidade pública, sendo 11 do sexo feminino e nove do sexo masculino, com idades entre 19 e 34 anos. Um dos participantes do sexo masculino não completou todas as etapas do procedimento, tendo sido excluído da pesquisa.
O critério de seleção dos participantes consistiu em estarem namorando há, pelo menos, seis meses, ininterruptamente, e não terem qualquer tipo de experiência com o tema "equivalência de estímulos". Com a finalidade de evitar a interferência de variáveis estranhas ao experimento, optou-se por não permitir a coleta de dados com as pessoas de um mesmo casal, sendo permitida a participação de apenas uma delas. 


\section{Local, materiais e instrumentos}

A coleta de dados foi realizada em uma sala reservada de um laboratório de pesquisa, medindo $2 \times 2 \mathrm{~m}$, contendo uma mesa e uma cadeira. Foi utilizado, para a coleta de dados, um microcomputador de mesa, rodando o programa Equivalência (Capocio, 2008). Um questionário de dados pessoais, confeccionado em duas versões (masculina e feminina), foi usado para obter informações sobre a duração do atual namoro e o nome do namorado(a).

A ECR, desenvolvida e validada para a população brasileira por Ramos et al. (1994), também foi usada nesse estudo. A ECR apresenta uma lista de sentenças que especificam situações de relacionamento, como: "Trocar o meu nome pode ser uma questão de esquecimento"; "Acho ruim quando ele fala que já teve momentos muito bons com outra pessoa". Ao responder, o participante deve apontar sua opinião a respeito de possíveis comportamentos de seu companheiro, colocando entre os parênteses ao lado da sentença o número que melhor representa seu julgamento: (1) Discordo completamente; (2) Discordo; (3) Em dúvida; (4) Concordo; (5) Concordo plenamente. Para o presente estudo foram aplicadas 20 das 32 sentenças da ECR original: Questões 2, 3, 5, 7, 8, 12, 13, 15, 16, 18, 19, 20, 22, 24,
$25,27,28,30,31,32$, selecionadas pelo fato de serem as mais apropriadas para o contexto da pesquisa e para a faixa etária dos(as) participantes. Por exemplo, foram excluídas sentenças como: "Em uma boate, é tolerável ele receber um recado pelo alto-falante: Você está demais hoje, quero falar com você". Também não foram usadas as questões que as instruções de correção de Ramos et al. (1994) recomendam excluir na pontuação do grau de ciúme. Os autores da ECR foram consultados com relação à retirada desse conjunto de questões e afirmaram que essa retirada não produziria alteração importante no escore obtido. O grau de ciúme (ou classificação) foi considerado 'Baixo' quando o escore obtido pelo participante era igual ou maior que 3,5; 'Moderado' quando estava entre 3,4 e 2,05; e 'Intenso' quando era igual ou menor que 2,04 .

Uma lista de nomes próprios foi usada para os participantes escolherem nomes de homens e de mulheres que não eram conhecidos por eles. Os estímulos usados como estímulos-modelo e/ou de comparação nas sessões de treino e de teste das relações condicionais para os participantes do sexo masculino estão na parte superior da Tabela $1 \mathrm{e}$, para as participantes do sexo feminino, na parte inferior dessa tabela. Os estímulos não familiares são os mesmos usados no estudo de Haydu et al. (2015).

Tabela 1

Estímulos Apresentados aos Participantes de Ambos os Sexos como Estímulos-Modelo ou de Comparação. Os Números Indicam as Classes de Estímulos a Serem Formadas e as Letras, o Conjunto de Estímulos que Compõem as Classes

\begin{tabular}{|c|c|c|c|c|c|}
\hline \multirow{2}{*}{ Sexos } & \multirow{2}{*}{ Estímulos } & \multicolumn{4}{|c|}{ Classes } \\
\hline & & 1 & 2 & 3 & 4 \\
\hline \multirow{3}{*}{ Masculi } & A & Nome do participante & $\begin{array}{l}\text { Nome de homem } \\
\text { desconhecido }\end{array}$ & $\begin{array}{l}\text { Nome de mulher } \\
\text { desconhecida }\end{array}$ & $\begin{array}{l}\text { Nome de homem } \\
\text { desconhecido }\end{array}$ \\
\hline & $\mathrm{B}$ & & & & \\
\hline & $\mathrm{D}$ & $\begin{array}{l}\text { Nome de mulher } \\
\text { desconhecida }\end{array}$ & $\begin{array}{l}\text { Nome de mulher } \\
\text { desconhecida }\end{array}$ & $\begin{array}{l}\text { Nome de homem } \\
\text { desconhecido }\end{array}$ & Nome da namorada \\
\hline \multirow{4}{*}{ Femin } & A & Nome da participante & $\begin{array}{l}\text { Nome de homem } \\
\text { desconhecido }\end{array}$ & $\begin{array}{l}\text { Nome de mulher } \\
\text { desconhecida }\end{array}$ & $\begin{array}{l}\text { Nome de mulher } \\
\text { desconhecida }\end{array}$ \\
\hline & B & & & & \\
\hline & $\mathrm{C}$ & & & & \\
\hline & $\mathrm{D}$ & $\begin{array}{l}\text { Nome de homem } \\
\text { desconhecido }\end{array}$ & $\begin{array}{l}\text { Nome de mulher } \\
\text { desconhecida }\end{array}$ & $\begin{array}{l}\text { Nome de homem } \\
\text { desconhecido }\end{array}$ & Nome do namorado \\
\hline
\end{tabular}




\section{Procedimento}

Os estudantes foram convidados a participar da pesquisa por meio de contato estabelecido em sala de aula por uma das pesquisadoras e, posteriormente, solicitados a confirmar o horário da visita ao laboratório por e-mail ou por telefone. Ao comparecerem ao laboratório, os participantes receberam informações gerais sobre a pesquisa e aqueles que aceitaram participar receberam o Termo de Consentimento Livre e Esclarecido, aprovado pelo Comitê de Ética em Pesquisa (Protocolo $n^{\circ}$ 028/12) da instituição dos autores, para ler e assinar, caso concordassem. Foi informado aos participantes que o estudo tratava de relações afetivas, e que maiores informações e detalhes poderiam ser obtidos ao final da coleta de dados de todos os participantes, não tendo sido mencionada a palavra "ciúme" em momento algum. Em seguida, os participantes receberam o questionário sobre dados pessoais para preencher e a lista de nomes próprios para assinalar aqueles que não eram nomes de pessoas que eles conhecessem. A seguinte instrução foi fornecida: Na tabela abaixo, selecione os nomes de homens e os nomes de mulheres que não correspondam a pessoas conhecidas por você. Finalmente, era agendada a próxima sessão, na qual os participantes eram expostos às tarefas no computador e respondiam à ECR.

O protocolo de treino e teste das relações entre estímulos programado no computador era personalizado já que os nomes selecionados pelo participante eram utilizados como sendo os estímulos A1, A2, A3, A4 e D1, D2, D3, D4 (ver Tabela 1). Os treinos e testes foram realizados da mesma maneira para homens e mulheres, com a diferença apenas das alterações dos estímulos "nome do namorado(a)", "nome do(a) participante", e na posição dos estímulos "nome de homem desconhecido", e "nome de mulher desconhecida". $\mathrm{O}$ arranjo de treino era do tipo linear, com o treino das relações condicionais $\mathrm{A} 1 \mathrm{~B} 1, \mathrm{~B} 1 \mathrm{C} 1, \mathrm{C} 1 \mathrm{D} 1, \mathrm{~A} 2 \mathrm{~B} 2, \mathrm{~B} 2 \mathrm{C} 2$, C2D2, A3B3, B3C3, C3D3, A4B4, B4C4, C4D4, e os testes das relações emergentes derivadas desse treino prévio. Os treinos e testes eram executados por meio do procedimento de MTS, em que o estímulo-modelo aparecia no centro da tela do monitor e os estímulos de comparação nos quatro cantos, ao redor do estímulo-modelo. Imediatamente após clicar sobre o estímulo-modelo, esse permanecia na tela e os quatro estímulos de comparação eram apresentados. As relações condicionais a serem ensinadas eram exibidas de forma aleatória nos diferentes blocos de treinos e nos blocos de testes. $\mathrm{O}$ treino de relações era do tipo misto, no qual todas as relações condicionais eram ensinadas no mesmo bloco. A Tabela 2 apresenta a sequência das etapas do procedimento, as relações ensinadas ou testadas em cada etapa e o número de tentativas nos blocos.

$\mathrm{Na}$ Etapa 1 (Treino Misto), as relações condicionais AB, $\mathrm{BC}$ e CD eram treinadas em blocos de 48 tentativas. Em cada uma das tentativas havia um estímulo de comparação positivo (aquele que estava de acordo com as classes especificadas pelos experimentadores) e três estímulos de comparação negativos (aqueles que estavam em desacordo com as classes especificadas pelos experimentadores), sendo variada a posição dos estímulos de comparação nas sucessivas tentativas. As seguintes instruções eram lidas para os participantes no início dessa etapa, realizada no computador, as quais eram semelhantes às do estudo de Haydu et al. (2015).

Sua tarefa é relacionar estímulos que aparecerão na tela do computador. O estímulo que aparecerá no centro da tela é o estímulo-modelo e os que aparecerão ao redor são os estímulos de comparação. Você deve clicar com o mouse sobre o estímulo de comparação que você considere estar relacionado com o estímulo-modelo que estiver no centro da tela. Somente um estímulo de comparação pode ser relacionado a cada estímulo-modelo e você deverá descobrir quais são os pares corretos. Você passará por algumas fases e só avançará quando atingir um determinado critério de acertos. Ao término do experimento, se você quiser, poderá obter maiores informações sobre os resultados, mas durante o procedimento, nenhuma informação adicional poderá ser fornecida. É muito importante que, durante a condução de nosso experimento, você não comente com outras pessoas sobre o procedimento da pesquisa ou sobre os estímulos que você verá. Não anote esses estímulos de nenhuma forma.

Tabela 2

Distribuição das Etapas do Procedimento com Identificação dos Blocos de Treino e de Testes, das Relações Ensinadas elou Testadas e o Número de Tentativas em Cada Bloco

\begin{tabular}{|c|c|c|c|}
\hline Etapas & Procedimento & Relações & Número de Tentativas \\
\hline 1 & Treino Misto & $\begin{array}{l}\mathrm{A} 1 \mathrm{~B} 1, \mathrm{~B} 1 \mathrm{C} 1, \mathrm{C} 1 \mathrm{D} 1, \mathrm{~A} 2 \mathrm{~B} 2, \mathrm{~B} 2 \mathrm{C} 2, \mathrm{C} 2 \mathrm{D} 2, \\
\mathrm{~A} 3 \mathrm{~B} 3, \mathrm{~B} 3 \mathrm{C} 3, \mathrm{C} 3 \mathrm{D} 3, \mathrm{~A} 4 \mathrm{~B} 4, \mathrm{~B} 4 \mathrm{C} 4, \mathrm{C} 4 \mathrm{D} 4\end{array}$ & 48 \\
\hline 2 & Teste de Linha de Base & $\begin{array}{l}\mathrm{A} 1 \mathrm{~B} 1, \mathrm{~B} 1 \mathrm{C} 1, \mathrm{C} 1 \mathrm{D} 1, \mathrm{~A} 2 \mathrm{~B} 2, \mathrm{~B} 2 \mathrm{C} 2, \mathrm{C} 2 \mathrm{D} 2, \\
\mathrm{~A} 3 \mathrm{~B} 3, \mathrm{~B} 3 \mathrm{C} 3, \mathrm{C} 3 \mathrm{D} 3, \mathrm{~A} 4 \mathrm{~B} 4, \mathrm{~B} 4 \mathrm{C} 4, \mathrm{C} 4 \mathrm{D} 4\end{array}$ & 24 \\
\hline 3 & Teste de Simetria & $\begin{array}{l}\mathrm{B} 1 \mathrm{~A} 1, \mathrm{C} 1 \mathrm{~B} 1, \mathrm{D} 1 \mathrm{C} 1, \mathrm{~B} 2 \mathrm{~A} 2, \mathrm{C} 2 \mathrm{~B} 2, \mathrm{D} 2 \mathrm{C} 2, \\
\mathrm{~B} 3 \mathrm{~A} 3, \mathrm{C} 3 \mathrm{~B} 3, \mathrm{D} 3 \mathrm{C} 3, \mathrm{~B} 4 \mathrm{~A} 4, \mathrm{C} 4 \mathrm{~B} 4, \mathrm{D} 4 \mathrm{C} 4\end{array}$ & 24 \\
\hline 4 & $\begin{array}{l}\text { Teste de Transitividade e } \\
\text { Transitividade Simétrica }\end{array}$ & $\begin{array}{l}\mathrm{A} 1 \mathrm{C} 1, \mathrm{~A} 1 \mathrm{D} 1, \mathrm{~B} 1 \mathrm{D} 1, \mathrm{C} 1 \mathrm{~A} 1, \mathrm{D} 1 \mathrm{~A} 1, \mathrm{D} 1 \mathrm{~B} 1 \\
\mathrm{~A} 2 \mathrm{C} 2, \mathrm{~A} 2 \mathrm{D} 2, \mathrm{~B} 2 \mathrm{D} 2, \mathrm{C} 2 \mathrm{~A} 2, \mathrm{D} 2 \mathrm{~A} 2, \mathrm{D} 2 \mathrm{~B} 2 \\
\mathrm{~A} 3 \mathrm{C} 3, \mathrm{~A} 3 \mathrm{D} 3, \mathrm{~B} 3 \mathrm{D} 3, \mathrm{C} 3 \mathrm{~A} 3, \mathrm{D} 3 \mathrm{~A} 3, \mathrm{D} 3 \mathrm{~B} 3 \\
\mathrm{~A} 4 \mathrm{C} 4, \mathrm{~A} 4 \mathrm{D} 4, \mathrm{~B} 4 \mathrm{D} 4, \mathrm{C} 4 \mathrm{~A} 4, \mathrm{D} 4 \mathrm{~A} 4, \mathrm{D} 4 \mathrm{~B} 4\end{array}$ & 48 \\
\hline
\end{tabular}


As respostas corretas foram todas seguidas por consequência visual que informou o acerto (Você acertou!), enquanto as que não estavam de acordo com o modelo foram seguidas por consequência visual que informava o erro (Você errou!). Um critério de $90 \%$ de acertos foi especificado para que o participante pudesse iniciar a etapa seguinte. Para os participantes que não atingiram esse critério, o procedimento foi repetido, na mesma sessão e sem interrupção, até que o critério fosse alcançado ou até que a sessão extrapolasse o limite de $1 \mathrm{~h}$. Caso o participante extrapolasse esse limite de tempo sem atingir o critério, ele era informado de que o experimento estava encerrado e os experimentadores agradeciam-no pela participação.

A partir da Etapa 2, foram realizados os testes compostos de blocos que eram apresentados apenas uma vez. Nessa etapa, não foi aplicado qualquer critério de acertos e as respostas não produziam consequências. $\mathrm{Na}$ Etapa 2, foi realizado o Teste de Linha de Base (teste das relações ensinadas $\mathrm{AB}, \mathrm{BC}$ e $\mathrm{CD}$ ), com 24 tentativas aleatórias (duas de cada tipo de relação). No início dessa etapa, a seguinte instrução foi lida: A partir de agora você não receberá mais nenhuma mensagem sobre o seu desempenho, indicando acerto ou erro. Mesmo assim, continue respondendo de acordo com o que você aprendeu.

$\mathrm{Na}$ Etapa 3 (Teste de Simetria), foram testadas as relações BA, CB, DC, em um único bloco com 24 tentativas aleatórias (duas de cada tipo de relação). Na Etapa 4 (Teste de Transitividade e Transitividade Simétrica), as relações $\mathrm{AC}, \mathrm{AD}, \mathrm{BD}, \mathrm{CA}, \mathrm{DA}, \mathrm{DB}$ foram testadas em um único bloco com 48 tentativas (duas de cada tipo de relação). Após a Etapa 4, cada participante recebeu a ECR para responder por escrito na mesma sala em que foram realizadas as tarefas no computador. Após o preenchimento da ECR, foi feito o agradecimento e marcado um encontro para que os participantes pudessem conhecer os resultados da pesquisa.

\section{RESULTADOS}

Dezenove participantes completaram todo o procedimento, sendo 11 do sexo feminino e oito do sexo masculino, identificados na primeira e segunda coluna da Tabela 3 . Dezessete participantes realizaram todas as etapas do procedimento em uma única sessão, e dois participantes (P6 e P7) o fizeram em duas sessões. Essa tabela apresenta a classificação e o escore de cada participante na ECR e as porcentagens de respostas corretas (que estavam de acordo com as relações especificadas pelos experimentadores) nos testes das relações emergentes (testes realizados nas Etapas 3 e 4). As porcentagens de acertos no Teste de Equivalência Global referem-se às 72 relações dos Testes de Simetria, Transitividade e Transitividade Simétrica (devido a uma falha de programação, o P17 não foi exposto ao teste de simetria, tendo realizado o Teste de Linha de Base duas vezes). As porcentagens de acertos nas relações condicionais A1D1 e D1A1, A2D2 e D2A2, A3D3 e D3A3, e A4D4 e D4A4 foram obtidas nos Testes de Transitividade e de Transitividade Simétrica.

Verifica-se, na Tabela 3, que quatro dos 19 participantes (P16, P17, P18 e P19) revelaram, por meio da ECR, ter grau de ciúme baixo e apresentaram de $97,9 \%$ a $100 \%$ de acertos no Teste de Equivalência Global. Todos os quatro formaram classes de equivalência de acordo com um critério de $90 \%$ de acertos. Os demais participantes (15) mostraram grau de ciúme moderado. Com relação à porcentagem de acertos no Teste de Equivalência Global, seis desses participantes (P1, P2, P3, P4, P5 e P13) não formaram as classes de equivalência de acordo com o critério, e dentre esses seis, quatro (P1, P2, P3, P4) são os participantes com escores mais baixos na ECR (indicador de maior grau de ciúme).

A comparação das porcentagens de acertos no Teste de Equivalência Global com os escores obtidos na ECR, feita por meio do cálculo do Índice de Correlação de Spearman, revelou correlação estatisticamente significante entre esses dados $(r=0,5282 ; p=0,020)$. Isso indica que os participantes que obtiveram escores mais altos na ECR (menor grau de ciúme) apresentaram porcentagens mais altas no Teste de Equivalência Global. Ainda, foram observadas correlações estatisticamente significativas entre os resultados da ECR e as porcentagens de acertos no teste das relações A1D1 e D1A1 ( $r=0,5452 ; \mathrm{p}=0,015)$, e A4D4 e D4A4 ( $\mathrm{r}=0,5404$; $\mathrm{p}=0,016)$. Os dados da ECR e a porcentagem de acertos no teste das relações A2D2 e D2A2 ( $r=0,4177 ; \mathrm{p}=0,079)$, e A3D3 e D3A3 (r=0,4210 p=0,072) não apresentam índices de correlação estatisticamente significativos. Pode-se observar, por meio de inspeção visual, que os participantes que apresentaram maiores escores na ECR (menor ciúme) apresentaram $100 \%$ de acertos na maior parte dos testes de transitividade e transitividade simétrica das relações A1D1 e D1A1, A2D2 e D2A2, A3D3 e D3A3, e A4D4 e D4A4.

A comparação entre participantes do sexo masculino e do sexo feminino em relação ao escore obtido por eles na ECR feita por meio do teste Mann-Whitney revelou não haver diferença estatisticamente significativa $(U=27,5 ; p=0,18)$. $O$ desempenho dos participantes do sexo masculino e do sexo feminino no Teste de Equivalência Global, ao ser comparado por meio do teste Mann-Whitney, também não revelou diferença estatisticamente significativa $(U=24,5 ; p=0,116)$. Devido aos resultados do tratamento estatístico comparativo entre homens e mulheres não terem apontado diferenças significativas, os dados dos dois sexos não foram separados para análise do tempo de reação apresentada a seguir.

Na Figura 1 estão os tempos de reação médios (em segundos) nas tentativas com respostas corretas e nas tentativas com respostas incorretas no Teste de Transitividade 
Tabela 3

Distribuição da Classificação e dos Escores Obtidos na Escala de Ciúme Romântico (ECR) e Porcentagens de Acertos no Teste de Equivalência Global e nos Testes das relações A1D1, D1A1 e A4D4, D4A4

\begin{tabular}{|c|c|c|c|c|c|c|c|c|}
\hline Participantes & Sexo & ECR & & $\begin{array}{c}\text { Teste de } \\
\text { Equivalência } \\
\text { Global }\end{array}$ & $\begin{array}{l}\text { Teste Relações } \\
\text { A1D1 e D1A1 }\end{array}$ & $\begin{array}{l}\text { Teste Relações } \\
\text { A2D2 e D2A2 }\end{array}$ & $\begin{array}{l}\text { Teste Relações } \\
\text { A3D3 e D3A3 }\end{array}$ & $\begin{array}{l}\text { Teste Relações } \\
\text { A4D4 e D4A4 }\end{array}$ \\
\hline & & Classificação & Escores & \multicolumn{5}{|c|}{ Porcentagens de acertos } \\
\hline 1 & $\mathrm{~F}$ & Moderado & 2,15 & 73,8 & 75 & 50 & 100 & 0 \\
\hline 2 & $\mathrm{~F}$ & Moderado & 2,22 & 85,4 & 75 & 100 & 50 & 50 \\
\hline 3 & $\mathrm{~F}$ & Moderado & 2,25 & 66,0 & 0 & 50 & 100 & 100 \\
\hline 4 & M & Moderado & 2,35 & 54,6 & 25 & 75 & 50 & 50 \\
\hline 5 & M & Moderado & 2,5 & 62,9 & 100 & 25 & 25 & 75 \\
\hline 6 & $\mathrm{~F}$ & Moderado & 2,67 & 99,3 & 100 & 100 & 100 & 100 \\
\hline 7 & $\mathrm{~F}$ & Moderado & 2,72 & 100,0 & 100 & 100 & 100 & 100 \\
\hline 8 & M & Moderado & 2,8 & 95,4 & 100 & 100 & 83 & 100 \\
\hline 9 & $\mathrm{~F}$ & Moderado & 2,87 & 94,4 & 75 & 100 & 100 & 100 \\
\hline 10 & $\mathrm{~F}$ & Moderado & 2,95 & 100,0 & 100 & 100 & 100 & 100 \\
\hline 11 & $\mathrm{~F}$ & Moderado & 2,97 & 97,7 & 100 & 100 & 100 & 100 \\
\hline 12 & $\mathrm{~F}$ & Moderado & 3,02 & 100,0 & 100 & 100 & 100 & 100 \\
\hline 13 & M & Moderado & 3,22 & 83,1 & 100 & 83 & 100 & 100 \\
\hline 14 & $\mathrm{~F}$ & Moderado & 3,22 & 95,6 & 100 & 100 & 100 & 100 \\
\hline 15 & M & Moderado & 3,25 & 90,0 & 100 & 50 & 75 & 63 \\
\hline 16 & $\mathrm{~F}$ & Baixo & 3,62 & 100,0 & 100 & 100 & 100 & 100 \\
\hline 17 & M & Baixo & 3,87 & 97,9 & 100 & 100 & 100 & 100 \\
\hline 18 & M & Baixo & 4,02 & 98,6 & 100 & 100 & 100 & 100 \\
\hline 19 & M & Baixo & 4,15 & 99,3 & 100 & 100 & 100 & 100 \\
\hline
\end{tabular}

Obs. Devido a uma falha de programação, o P17 não foi exposto ao Teste de Simetria, tendo realizado o Teste de Linha de Base duas vezes. Assim, o cálculo da porcentagem global foi feito a partir dos dados dos Testes de Transitividade e de Transitividade Simétrica.

\section{$\square$ Acertos $⿴$ Erros}

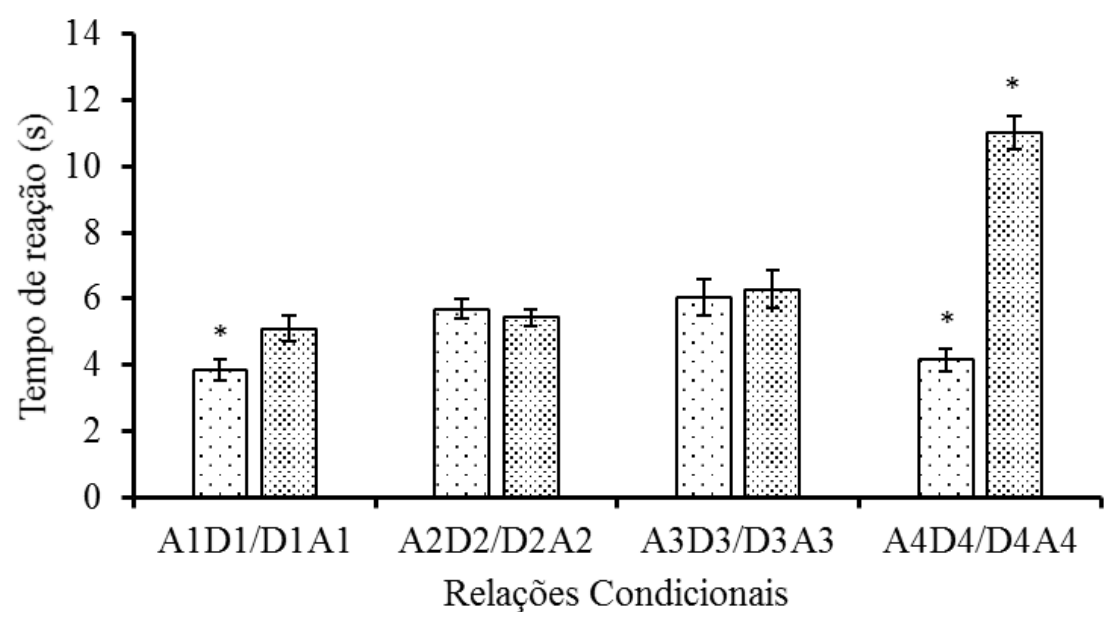

Figura 1. Tempo de reação médio (em segundos) nas tentativas com acertos e com erros nos testes das relações emergentes de transitividade (A1D1 - nome do participante/nome de mulher desconhecida ou nome da participante/nome de homem desconhecido, A2D2 - nome de homem desconhecido/nome de mulher desconhecida, A3D3 - nome de mulher desconhecida/nome de homem desconhecido, A4D4 - nome de homem desconhecido/nome da namorada ou nome de mulher desconhecida/nome do namorado) e de transitividade simétrica (D1A1 - nome de mulher desconhecida/nome do participante, D2A2 - nome de mulher desconhecida/nome de homem desconhecido, D3A3 - nome de homem desconhecido/nome de mulher desconhecida, D4A4 - nome da namorada/nome de homem desconhecido ou nome do namorado/nome de mulher desconhecida) das quatro classes de equivalência. O erro padrão da média está representado na figura pelas barras de erro inseridas em cada coluna. $\mathrm{O}$ asterisco indica diferença estatisticamente significativa. 
e de Transitividade Simétrica, considerando os dados das quatro classes em separado (A1D1/D1A1; A2D2/D2A2; A3D3/D3A3; A4D4/D4A4). A análise estatística, feita por meio do Teste Kruskal-Wallis, revelou que há diferença estatística significativa $(\chi 2=23,11 ; p=0,016)$. Na comparação dos pares post hoc feita por meio do Teste Dunn, verificou-se diferença estatisticamente significativa entre o tempo de reação nas tentativas com acertos das relações A4D4/D4A4 e nas tentativas com erros dessas mesmas relações. De forma geral, o tempo de reação foi maior nas tentativas com resposta incorretas do que naquelas com respostas corretas, exceto no caso das relações das classes A2D2/ D2A2, em que foram relacionados nomes de homens e mulheres desconhecidos. Entretanto, essa diferença é de apenas 0,3s. Nas tentativas com relações A1D1/D1A1 e A4D4/ D4A4, a diferença é maior (1,2 s e 6,9 s, respectivamente), sendo a maior diferença $(6,9 \mathrm{~s})$ observada nas tentativas da classe A4D4/D4A4, nas quais eram relacionados o nome do namorado(a) e o nome de uma mulher/homem desconhecido(a).

$\mathrm{Na}$ Figura 2 estão representadas as porcentagens médias de respostas incorretas na formação das relações condicionais entre os estímulos referentes às Classes 1, 2, 3 e 4 . Na parte superior dessa figura estão os resultados dos participantes do sexo masculino e, na parte inferior, do feminino. Para essa análise, os dados dos homens e das

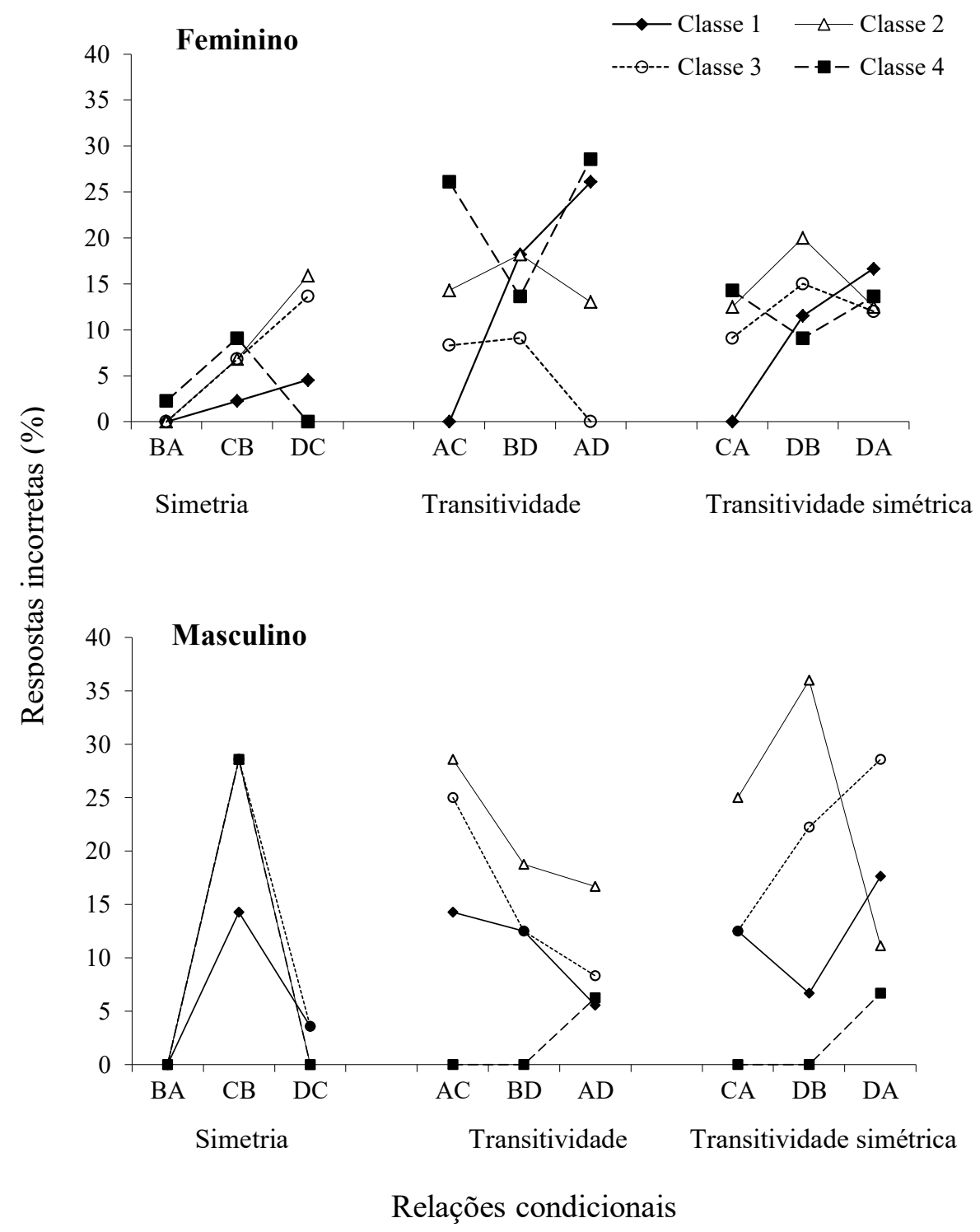

Figura 2. Porcentagens médias de respostas incorretas nos testes das relações condicionais de Simetria (BA, CB, DC), Transitividade (AC, BD, AD) e Transitividade Simétrica (CA, DB, DA). No painel superior estão os dados das participantes do sexo feminino e no painel inferior, os dados dos participantes do sexo masculino. 
mulheres foram apresentados separadamente devido aos diferentes padrões de respostas emergentes observados. Com relação aos homens, verifica-se que, no Teste de Simetria, as porcentagens mais altas de escolhas incorretas ocorreram nas tentativas das relações C1B1(14,29\%), C2B2 (28,6\%), C3B3 $(28,6 \%)$ e C4B4 $(28,6 \%)$ em comparação com as demais relações de simetria. Essas relações (CB) são as que envolviam duas figuras. Nas tentativas de Teste de Simetria da relação BA das quatro classes e as relações D4C4, não ocorreram erros. No Teste de Transitividade, as porcentagens mais altas de respostas incorretas ocorreram nas tentativas de teste das relações AC das Classes 1, 2 e 3 (A1C1 - 14,29\%; A2C2 - 28,6\%; $\mathrm{A} 3 \mathrm{C} 3-25 \%$ ). Essas tentativas envolviam relações entre um nome de homem/mulher desconhecido(a) e uma figura. No Teste de Transitividade Simétrica, as porcentagens mais altas de erros ocorreram nas tentativas de teste das relações das Classes 2 e 3 (C2A2 - 25\%; D2B2 - 36\%; D3B3 $-22,2 \%$ ), que envolviam um nome de homem/mulher desconhecido(a) e uma figura; e no teste da relação D3A3 $(28,6 \%)$, que envolvia um nome de homem desconhecido e um nome de uma mulher desconhecida. No geral, a classe com menores porcentagens de respostas incorretas foi a Classe 4: A4B4 - 5,56\% (relação entre nome de mulher e figura), C4B4 - 28,6\% (relação entre duas figuras), A4D4 $-6,3 \%$ (relação entre nome de homem desconhecido e o nome da namorada e D4A4 - 6,7\% (relação entre o nome do namorado e de mulher desconhecida).

Com relação às mulheres, observa-se que, nos testes de simetria, as porcentagens de erros, no geral, foram mais baixas em comparação aos demais testes. As maiores porcentagens de erros ocorreram nas tentativas de testes das relações D2C2 (15,91\%) e D3C3 (13,64\%), que envolviam duas figuras. Nos testes das relações de transitividade, as porcentagens de respostas incorretas mais altas ocorreram nas tentativas de teste das relações da Classe 1 (A1D1 $26,09 \%$ ), que envolvia o nome da participante e um nome de homem desconhecido, e da Classe 4 (A4C4 - 26,09\%; A4D4-28,57\%) que envolvia o nome do namorado e um nome de mulher desconhecida. No Teste de Transitividade Simétrica, as porcentagens de erros mais altas ocorreram nas tentativas de teste das relações C4A4 - 14,29\% (relação entre figura e nome de mulher desconhecida), D2B2 - 20\% (relação entre nome de mulher desconhecida e figura), D3B3 - 15\% (relação entre nome de homem desconhecido e figura) e D1A1 - 16,67\% e D4A4 - 13,64\% (relações entre nome do namorado e de mulher desconhecida).

\section{DISCUSSÃo}

O presente estudo visou replicar o procedimento do estudo de Haydu et al. (2015), tendo como objetivo avaliar o efeito de estímulos com alto grau afetivo préexperimentalmente constituído (ciúme inferido a partir do relato dos participantes na ECR) na formação de classes de estímulos equivalentes. No presente estudo, ampliou-se o número de participantes do sexo masculino, uma vez que, no estudo de Haydu et al., havia apenas um estudante do sexo masculino, que foi justamente o único a apresentar escore de ciúme baixo. Uma modificação foi introduzida no presente estudo quanto ao tipo de estímulo de uma das classes a serem formadas, que consistiu da inclusão do nome do próprio participante como um dos estímulos de treino e de teste, sendo que o nome do participante, de acordo com o treino de equivalência de estímulos, nunca seria relacionado ao nome do namorado(a) e sim a um nome desconhecido, da mesma forma que o nome do namorado(a) deveria ser relacionado a um nome desconhecido. Além disso, o número de tentativas de teste das relações de transitividade e de transitividade simétrica foi aumentado para que todas as relações condicionais fossem testadas mais de uma vez.

No Teste de Equivalência Global, 13 dos 19 participantes atingiram o critério de $90 \%$ de acertos. Por falha de programação, um dos participantes (P17) não foi submetido ao Teste de Simetria. O desempenho desse participante mostrou que essa falha não prejudicou a formação das classes de equivalência, uma vez que ele atingiu um índice de $97,9 \%$ de acertos no Teste de Equivalência Global.

Todos os quatro participantes classificados como tendo baixo grau de ciúme atingiram o critério de formação de classes, apresentando $100 \%$ de acertos nos testes das relações emergentes que envolviam os nomes do namorado e/ou o próprio nome. Os 10 participantes com graus de ciúme moderado (escore entre 2,67 e 3,25) também formaram as classes de equivalência, atingindo o critério de $90 \%$. Os demais participantes (5 dos 19), aqueles que apresentaram os escores mais baixos na ECR (maior grau de ciúme), não atingiram o critério de $90 \%$ de acertos no Teste de Equivalência Global, sendo três do sexo feminino e dois do sexo masculino. Esses resultados permitem sugerir que, no caso desses últimos participantes (com maior grau de ciúme), a função afetiva do estímulo, pré-experimentalmente estabelecida e mensurada por meio da ECR, prejudicou a emergência das classes de equivalência estabelecidas pelos experimentadores, o que corrobora os achados de Haydu et al. (2015), e estão de acordo com outros estudos da literatura (e.g., Plaud, 1995; Watt, Keenan, Barnes, \& Cairns, 1991; Dack, McHugh, \& Reed, 2012; Merwin \& Wilson, 2005). Cabe destacar que não só as relações inconsistentes com a história pré-experimental não foram estabelecidas entre os participantes com escores mais baixos na ECR (alto grau de ciúme), mas as classes de equivalência não foram formadas. Isso possibilita levantar a hipótese de que tenha havido 
transferência de função para estímulos que não aqueles com supostas funções emocionais pré-experimentais. Essa hipótese, no entanto, precisaria ser investigada, o que poderia ser feito por meio de registros de respostas eletrofisiológicas, como no estudo desenvolvido por Valverde, Luciano e Barnes-Holmes (2009). No estudo de Haydu et al. (2015), cinco participantes apresentaram escores com valores de ciúme intenso e apenas um com ciúme baixo e, no presente estudo, nenhum participante foi classificado com ciúme intenso e quatro foram classificados com ciúme baixo. Essa diferença, no entanto, não prejudicou a comparação dos resultados obtidos nesses dois estudos. Em ambos, quanto menor o escore obtido na ECR (maior o grau de ciúme), menor foi a probabilidade de emergiram classes de equivalência. A substituição de um dos estímulos pelo nome do participante em uma das classes a serem formadas (Classe 1), permitiu observar que o efeito de interferência (a não formação da relação condicional) ocorreu mesmo na relação em que o ciúme não estava presente, mas havia inconsistência com a história de reforço no contexto pré-experimental dos participantes. Isto é, relacionar o nome do participante ao nome de um(a) homem/mulher desconhecido(a) estava em contradição ao que é feito no dia a dia, uma vez que nesse contexto, o nome do(a) namorado(a) é relacionado com o nome do(a) participante.

No presente estudo, dentre os participantes que apresentaram os menores graus de ciúme (escores abaixo de 2,5 na ECR) e formaram as classes de equivalência de acordo com o critério de $90 \%$ de acertos, observou-se uma exceção, que foi o resultado do P13. Esse participante atingiu $100 \%$ de acertos nos testes das relações A1D1/ D1A1 e A4D4/D4A4 (ver Tabela 3), mas, no Teste de Equivalência Global, a porcentagem ficou em $83,1 \%$, tendo apresentado respostas incorretas em testes das relações das Classes 2 e 3. As Classes 1 e 4 podem ter sido formadas com uma probabilidade maior $(100 \%$ de acertos) porque envolviam nomes familiares - o nome do participante e o nome da namorada, além de serem estímulos nomeáveis. A facilitação no estabelecimento de relações de equivalência que envolviam estímulos familiares (nomes, figuras, letras, sílabas) e estímulos nomeáveis foi amplamente estudada e demonstrada em uma série de estudos, como no de Arntzen e Lian (2010), Fields, Arntzen, Nartey e Eilifsen (2012) e O'Connor, Rafferty, Barnes-Holmes e Barnes-Holmes (2009).

No estudo desenvolvido por Arntzen e Lian (2010), foi demonstrado que houve diferença na formação de classes de equivalência quando estímulos familiares (figuras) foram usados como nódulos na formação de três classes de equivalência com três membros cada uma, em comparação à formação de classe tendo estímulos abstratos como nódulos. Nesse estudo, a variável independente foi manipulada em diferentes fases. Um grupo foi submetido primeiramente ao procedimento com os estímulos familiares, enquanto o outro grupo foi submetido ao procedimento com os estímulos abstratos, havendo a reversão dessas condições na fase seguinte. Delineamentos como estes são comumente usados na literatura da área, ao passo que, no presente estudo, diferentemente, foi feita uma comparação entre as classes estabelecidas simultaneamente com estímulos que apresentavam diferentes valências. Essa diferença de procedimento e na forma de análise dos dados possibilitou observar que houve facilitação na formação das relações de equivalência que envolviam estímulos familiares e/ou nomeáveis, mas que quando era necessária a emissão de respostas de escolha que envolviam relações inconsistentes com a história pré-experimental, a porcentagem de respostas erradas foi maior do que nas demais condições. Esse efeito é claramente observado nos resultados dos participantes do sexo masculino. Por exemplo, os dados referentes à Classe 4 indicam que os participantes não apresentaram respostas incorretas nos Testes de Simetria, exceto na condição em que eles tiveram que relacionar duas figuras não familiares (relação B4C4). Nos Testes de Transitividade e de Transitividade Simétrica, os participantes não apresentaram respostas incorretas nas tentativas de teste das relações que envolviam pelo menos um estímulo nomeável (A4C4 e B4C4 - nome de homem/mulher desconhecido(a), nome do(a) participante ou o próprio nome). Entretanto, quando a relação era inconsistente com a história experimental (A4D4 e D4A4), a porcentagem de erros foi maior do que nos testes das demais relações dessa classe. Um efeito igual foi observado no Teste de Transitividade Simétrica da Classe 1 (D1A1), em que um dos estímulos era o nome do participante e o outro o nome de mulher desconhecida.

Resultado semelhante ao descrito no parágrafo anterior foi obtido com as participantes do sexo feminino em relação às Classes $1 \mathrm{e} 4$, as quais apresentaram um maior número de respostas incorretas nas tentativas de teste das relações que envolviam um nome de mulher e o nome do namorado (A4D4), e nas que envolviam o próprio nome e o nome de um homem desconhecido (A1D1 e D1A1), mas a porcentagem de erros foi um pouco menor nos testes das relações D4A4 do que C4A4. Ao se considerar os dados dos participantes do sexo feminino e do sexo masculino, de forma geral, observou-se que, nos testes das relações de transitividade e transitividade simétrica, as porcentagens de respostas incorretas foram menores nas tentativas em que as relações envolviam dois nomes do que naquelas que envolviam um nome e uma figura não familiar (Classes 2 e 3). Por outro lado, nas tentativas de teste que envolviam relações das Classes 1 e 4 , as porcentagens de erros foram maiores nas tentativas que envolvem dois nomes. As únicas exceções dentre os 16 conjuntos de testes (Teste de Transitividade e de Transitividade Simétrica das quatro classes, dos participantes do sexo masculino e do feminino) foram os resultados referentes à Classe 1, no Teste de Transitividade, e à Classe 3, no Teste de Transitividade Simétrica, dos participantes do sexo masculino, e os resultados da Classe 3 (D3B3>D3A3) dos participantes do sexo feminino. Resumindo, tem-se que 
seis dos 16 conjuntos de testes mostraram efeito de facilitação pela familiaridade e/ou nomeabilidade dos estímulos, e sete demostraram que a função afetiva do estímulo, préexperimentalmente estabelecida, prejudicou a emergência das relações condicionais. Os resultados do presente estudo permitem concluir que a função dos estímulos estabelecida na história pré-experimental (inferida a partir dos dados da ECR) esteve correlacionada com a emergência de relações de equivalência dos participantes. Quatro dos 19 participantes não formaram as classes de equivalência, tendo sido afetadas principalmente aquelas relações que poderiam ser as mais facilmente formadas por envolverem pares de nomes de pessoas de sexo oposto (nomeáveis e familiares). Além disso, pode-se concluir que a familiaridade dos nomes apresentados como estímulos a serem relacionados e a possibilidade de nomeá-los contribuiu para facilitar a emergência de relações condicionais. $\mathrm{Na}$ comparação dos resultados dos participantes do sexo masculino e do sexo feminino, não se obteve diferenças estatisticamente significantes entre os escores obtidos por homens e mulheres na ECR, assim como nos Testes de Equivalência Global. Entretanto, o número de respostas incorretas apresentado pelos participantes do sexo feminino nos testes das relações emergentes, em comparação aos participantes do sexo masculino, foi bem maior diante das relações que envolviam relações inconsistentes com a história pré-experimental. Esse fato sugere que esses dois grupos comportaram-se de forma diferente referente a esse aspecto.

A análise dos efeitos de funções de estímulos estabelecidas na história pré-experimental sobre a formação e a modificação de relações de equivalência se caracteriza como um método importante para investigar experimentalmente comportamentos e temas difíceis de serem acessados pelo experimentador em laboratórios, como o preconceito racial e religioso, a ansiedade, o abuso sexual, o jogo patológico, o ciúme romântico, dentre outros. A relevância do tema, bem como a variabilidade nos resultados obtidos, o número alto de erros em classes que não envolvem os estímulos com funções eliciadoras estabelecidas pré-experimentalmente e a amplitude de variáveis envolvidas, indica ser necessário que mais estudos sejam realizados para que possam ser controladas variáveis como as características dos estímulos usados, a estrutura de treino, o MTS simultâneo versus MTS com atrasado, o número de estímulos e de classes a serem formadas, a idade e o sexo dos participantes, e a manipulação da história experimentalmente construída. A compreensão do efeito dessas variáveis permitirá estruturar um modelo de análise e de avaliação clínica, bem como outros tipos de análises e de métodos de avaliação.

\section{REFERÊNCIAS}

Adcock, A. C., Merwin, R. M., Wilson, K. G., Drake, C. E., Tucker, C. I., \& Elliott, C. (2010). The problem is not learning: Facilitated acquisition of stimulus equivalence classes among low achieving college students. The Psychological Record, 60, 43-56.

Arntzen, E., \& Lian, T. (2010). Trained and derived relations with pictures versus abstract stimuli as nodes. The Psychological Record, 60, 659-677.

Buss, D. B. (1994). The evolution of desire: Strategies of human mating. New York: Basic Books.

Capocio, V. P. (2008). Software Equivalência [Programa de computador]. Ribeirão Pires, SP.

Clayton, M. C., \& Hayes, L. J. (2007). Using stimulus equivalence to demonstrate pre-existing linguistic relations: A systematic replication and extension. Revista Mexicana de Análisis de la Conducta, 33, 225-238. doi: 10.5514/rmac.v33.i2.16257

de Carvalho, M. P., \& de Rose, J. C. (2014). Understanding racial attitudes through the stimulus equivalence paradigm. The Psychological Record, 64, 527-536.

Costa, N. (2009). Busca de definição operacional de ciúme: Uma construção teórica e empírica (Tese de doutorado). Universidade Federal do Pará, Belém, Brasil.

Costa, N., Almeida, C., Gomes, H., Lobato, J., Gondim, L., Silva, M., ... Lima, V. A. (2014). O ciúme está relacionado ao amor: Contribuições de uma perspectiva analítico-comportamental. Perspectivas em Análise do Comportamento, 5, 40-48. Recuperado de http://pepsic.bvsalud.org/pdf/pac/v5n1/ v5n1a04.pdf

Dack, C., McHugh, L., \& Reed, P. (2012). Transfer of judgments of control to a target stimulus and to novel stimuli through derived relations. Learning and Behavior, 40, 448-464. doi: 10.3758/s13420-012-0066-6
Dougher, M. J. (1998). Stimulus equivalence and the untrained acquisition of stimulus functions. Behavior Therapy, 29, 577591. doi: 10.1016/S0005-7894(98)8001 8-X

Dougher, M. J., Augustson, E., Markham, M. R., Greenway, D. E., \& Wulfert, E. (1994). The transfer of respondent eliciting and extinction functions through stimulus equivalence classes. Journal of the Experimental Analysis of Behavior, 62, 331 -351. doi: 10.1901 /jeab.1 994.62-331

Dixon, M. R., Bihler, H. L., \& Nastally, B. L. (2011). Slot machine preferences of pathological and recreational gamblers are verbally constructed. The Psychological Record, 61, 93-112.

Dixon, M. R., \& Lemke, M. (2007). Reducing prejudice towards Middle Eastern persons as terrorists. European Journal of Behavior Analysis, 8, 5-12.

Fields, L., Arntzen, E., Nartey, R. K., \& Eilifsen, C. (2012). Effects of a meaningful, a discriminative, and a meaningless stimulus on equivalence class formation. Journal of the Experimental Analysis of Behavior, 97, 163-181. doi: 10.1901/jeab.2012.97-163.

Haydu, V. B., Gaça, L. B., Cognetti, N. P., Costa, C. E., \& Tomanari, G. Y. (2015). Equivalência de estímulos e ciúme: Efeito de história pré-experimental. Psicologia Reflexão e Crítica, 28, 490-499. doi: 10.1590/1678-7153.201528308

Keenan, M., McGlinchey, A., Fairhurst, C., \& Dillenburger, K. (2000). Accuracy of disclosure and contextual control in child abuse: Developing procedures within the stimulus equivalence paradigm. Behavior and Social Issues, 10, 1-17. doi: 10.5210/ bsi.v10i0.131

Leslie, J. C., Ulster-Jordanstown, U., Tierney, K. J., Robinson, C. P., \& Keenan, M. (1993). Differences between clinically anxious and non-anxious subjects in a stimulus equivalence training task involving threat words. Psychological Record, 43, 153-161. 
Merwin, R. M., \& Wilson, K. G. (2005). Preliminary findings on the effects of self-referring and evaluative stimuli on stimulus equivalence class formation. The Psychological Record, 55, 561-575.

Munnelly, A., Martin, G., Dack, C., Zedginidze, A., \& McHugh, L. (2014). The transfer of social exclusion and inclusion functions through derived stimulus relations. Learning \& Behavior, 42, 270-280. doi: 10.3758/s13420-014-0144-z

O’Connor, J., Rafferty, A., Barnes-Holmes, D., \& Barnes-Holmes, Y. (2009). The role of verbal behavior, stimulus nameability, and familiarity on the equivalence performances of autistic and normally developing children. The Psychological Record, $59,53-74$.

Plaud, J. J. (1995). The formation of stimulus equivalence classes: Fear-relevant and fear-irrelevant stimulus classes. The Psychological Record, 45, 207-222.

Plaud, J. J., Gaither, G. A., Franklin, M., Weller, L. A., \& Barth, J. (1998). The effects of sexually explicit words on the formation of stimulus equivalence classes. The Psychological Record, 48, 63-79.

Postalli, L. M. M., Schmidt, A, Nakachima, R. Y., \& de Souza, D. G. (2013). Controle instrucional e classes de estímulos equivalentes que incluem verbos e ações. Psicologia: Reflexão e Crítica, 26, 136-150. 2013. doi: 10.1590/S010279722013000100015

Ramos, A. L. M., Yazawa, S.A.K., \& Salazar, A. F. (1994). Desenvolvimento de uma escala de ciúme romântico. Psicologia: Teoria e Pesquisa, 10, 439-451.

Rehfeldt, R. A. (2011). Toward a technology of derived stimulus relations: An analysis of articles published in the Journal of
Applied Behavior Analysis, 1992-2009. Journal of Applied Behavior Analysis, 44, 109-19. doi: 10.1901/jaba.2011.44-109.

de Rose, J. C., \& Bortoloti, R. (2007). A equivalência de estímulos como modelo do significado. Acta Comportamentalia, 15, 83-102.

Santos, S. L., Mizael, T. M., \& de Rose, J. C. (2016). Contribuições do paradigma de equivalência de estímulos para o estudo das atitudes. Interação em Psicologia, 20, 124-134. doi: 10.5380/ psi.v20i2.46278

Sidman, M. (1990). Equivalence relations: Where do they come from? In H. Lejuene \& D. Blackman (Orgs.), Behavior analysis in theory and practice: Contributions and controversies (pp. 93-114). Hillsdale, NJ: Erlbaum.

Sidman, M. (1994). Equivalence relations: A research story. Boston: Authors Cooperative.

Sidman, M. (2000). Equivalence relations and the reinforcement contingency. Journal of the Experimental Analysis of Behavior, 74, 127-146. doi: 10.1901/jeab.2000.74-127.

Sidman, M., \& Tailby, W. (1982). Conditional discrimination vs. matching to sample: An expansion of the testing paradigm. Journal of the Experimental Analysis of Behavior, 37, 5-22. doi: 10.1901/jeab.1982.37-5

Valverde, M. R., Luciano, C., \& Barnes-Holmes, D. (2009). Transfer of aversive respondent elicitation in accordance with equivalence relations. Journal of the Experimental Analysis of Behavior, 92, 85-111. doi: 10.1901/jeab.2009.92-85

Watt, A., Keenan, M., Barnes, D., \& Cairns, E. (1991). Social categorization and stimulus equivalence. The Psychological Record, 41, 33-50.

Wulfert, E., Greenway, D. E., Farkas, P., Hayes, S. C. \& Dougher, M. J. (1994). Correlation between self-reported rigidity and 